Investigaciones Fenomenológicas, n. 8, 2011, 159-168.

e-ISSN: $1885-1088$

\title{
Cuerpo y fenomenología "asubjetiva" en Jan Patočka*
}

\author{
Iván Ortega Rodríguez \\ Universidad Pontificia Comillas, Madrid, España \\ ivan.ortega.rodriguez@gmail.com
}

\begin{abstract}
Resumen: En este trabajo describimos las líneas básicas del análisis fenómeno-lógico de la corporalidad en Patočka. El estudio de la corporalidad es un elemento clave en el proyecto de fenomenología asubjetiva de Jan Patočka. Es en ella donde se da la inserción del sujeto finito en el mundo y es en ella donde se hace comprensible el dinamismo del sujeto en el mundo, un sujeto que no "constituye" el mundo sino que es una fuerza en el campo de fuerzas del aparecer.
\end{abstract}

Palabras clave: Fenomenología, cuerpo, corporalidad, Jan Patočka.

\begin{abstract}
In this paper we describe the basic lines of Jan Patočka's analysis of embodiment. The study of corporeality is a key element in Patočka's project of an a-subjetive Phenomenology. It is within corporeality where the finite subject's insertion in the world takes place and it is there that the subject's dynamism becomes understandable; this subject does not "constitute" the world but is a force in the field of forces of appearance.
\end{abstract}

Keywords: Phenomenology, body, corporeality, Jan Patočka.

En este ensayo nos ocuparemos del filósofo checo Jan Patočka. Poco conocido aún en España, Patočka es sin embargo considerado como uno de los autores de referencia en la filosofía del siglo XX. Su pensamiento se estructura en filosofía primera y filosofía práctica, donde la fenomenología se articula con la filosofía de la historia, en una tensión ética fundamental. Sus posiciones fenomenológicas son base para su teoría sobre los movimientos de la existencia, que traslucen un impulso ético fundamental sobre el que se articulan todos los demás temas de su filosofía. Abordar una presentación global del pensamiento de Patočka, analizando las conexiones entre fenomenología y filosofía práctica, desborda los límites de esta contribución. El objetivo de este ensayo es hacer una breve presentación del pensamiento fenomenológico de Jan Patočka a partir de su intento más audaz. Nos centraremos en un conjunto de textos escritos

\footnotetext{
* Este artículo es parte de una investigación que culminó en una tesis doctoral con título Fenomenología, Filosofía de la historia y Política en Jan Patočka (Universidad Pontificia de Comillas, Madrid, 2011).
} 
al final de su vida, entre 1960 y 1976, donde explora las posibilidades de una refundación "asubjetiva" de la fenomenología ${ }^{1}$.

\section{LA CRÍTICA A LA FENOMENOLOGÍA TRANSCENDENTAL}

Jan Patočka busca superar el idealismo de la fenomenología transcendental de Husserl, pues a su juicio constituye ésta una desviación de la intención original de la fenomenología. Es sabido que Husserl fue derivando hacia el idealismo en la medida que consideró que el aparecer de los objetos y del mundo debía remitirse a un último horizonte subjetivo constituyente ante el cual tenían necesariamente que comparecer -siendo a su vez este horizonte intersubjetivo- con lo que se da una cierta "primordialidad" de lo subjetivo sobre lo objetivo. Patočka dedicó largas consideraciones a esta cuestión; y concluyó que un análisis fenomenológico verdaderamente radical no conduce a afirmar un horizonte subjetivo constituyente al que hubiera que remitir últimamente todo objeto en su aparecer y, con ello, el mundo en el que estos objetos aparecen y dentro del cual desarrollamos la vida. De acuerdo con Patočka, llegamos ciertamente a determinar un mundo en que se desarrolla la vida. Este mundo de la vida es analizable en sus estructuras, y ciertamente es el punto de partida para toda labor teórica. Sin embargo, este mundo no es en absoluto un mundo constituido por una conciencia "previa" y que sea en cierto modo su a priori. Todo lo contrario, afirma Patočka, es el mundo la condición previa para todo aparecer, $y$, sobre todo, para la realización de la intersubjetividad ${ }^{2}$.

Los caminos que Patočka recorre en su obra para desarrollar este tema de la primacía del mundo no siguen un esquema único, sino que se configuran como intentos varios, que permanecen en un estado de provisionalidad. En todos estos intentos, empero, podemos señalar, si nuestra interpretación es correcta,

\footnotetext{
${ }^{1}$ Por fortuna, una buena parte de estos textos está disponible en castellano, en la reciente compilación de Agustín Serrano de Haro, El movimiento de la existencia humana, Madrid, Encuentro, 2004. Otros textos se encuentran en una recopilación en francés titulada Qu'est-ce que la phénoménologie?, Grenoble, Jerôme Millon, 2002; compilación y traducción de Erika Abrams. En castellano también puede consultarse con provecho la traducción de unas lecciones de Patočka en la Univerzita Karlova de Praga (impartidas en el breve periodo en que pudo enseñar públicamente): Introducción a la fenomenología, Barcelona, Herder, 2005; traducción de Juan A. Sánchez, revisión de Iván Ortega. Además de introducir la obra de Husserl y la de Heidegger, añade Patočka comentarios que van en la línea de la fenomenología asubjetiva que aquí tratamos.

2 Jan Patočka, "La phénoménologie, la philosophie phénoménologique et les Méditations Cartésiennes de Husserl", en Qu'est-ce que la phénoménologie? Cfr. p. 159.
} 
un mismo patrón: el subjetivismo transcendental se debe a un añadido teórico no fenomenológico, esto conduce a que Husserl no ejecute una epojé verdaderamente radical, lo cual se debe a que da primacía a la necesidad de fundamentar absolutamente el conocimiento, así como a que considere que la salida al objetivismo de la razón científica está en afirmar la subordinación del mundo a la intersubjetividad en comunicación ("mónada intersubjetiva"). Sin embargo, afirma Patočka, una epojé radical muestra un aparecer de mundo que da fe de sí mismo desde sí mismo, que es además una totalidad previa para que todo aparecer se dé, que es previo al aparecer de este mismo mundo como "totalidad fáctica", y que es condición previa para la comunicación intersubjetiva y la constitución de la misma mónada intersubjetiva. Veamos esto un poco más de cerca.

Husserl tenía como máxima el "principio de todos los principios": tomar y analizar los datos sólo en lo que éstos dan desde sí mismos. Sin embargo, Patočka considera que Husserl introduce un elemento no justificado radicalmente. Hablamos de la conciencia tomada como un todo cerrado en sí, a modo de un ente o región de ser de un tipo especial. Es cierto que al analizar las vivencias, vemos que en ellas hay siempre una fundamental referencia a la subjetividad viva. No obstante, esto no significa que se nos muestre la subjetividad y la conciencia como un todo, y como un tipo de realidad con sus características definidas. Para Patočka, Husserl pasa sin más de la constatación de la vida subjetiva en toda vivencia a afirmar un tipo de ente unitario con unas características claramente definibles. Husserl habría realizado de este modo una "transposición de evidencias", por la que la esfera fenomenal, el aparecer mismo como siendo vivido $-\mathrm{y}$ del que ciertamente no cabe dudar-, se habría tomado como evidencia de la conciencia en tanto "ente subjetivo"3. Más aún, la consideración de la "esfera transcendental" como configurada en noesis y noema corresponde a una inadecuada descripción de la esfera fenomenal. Patočka cree que Husserl generaliza una clase particular de vivencias, pues la experiencia de la percepción dada como distinta de las sensaciones se convierte en modelo para toda cogitatio. Esto, afirma Patočka, conduce a la consabida división entre el polo subjetivo y el polo objetivo de la vivencia, que se generalizan y se consideran

\footnotetext{
${ }^{3}$ Cfr. Jan Patočka, "El subjetivismo de la fenomenología husserliana y la posibilidad de una fenomenología 'asubjetiva'", en El movimiento de la existencia humana, pp. 104-109.
} 
como polos de la totalidad unitaria y omnienglobante de la conciencia transcendental ${ }^{4}$.

De esta manera, según considera Patočka, Husserl introduce un concepto de conciencia derivado de la tradición cartesiana ${ }^{5}$, en el que la autoevidencia de la misma, captada en la autorreflexión, es un presupuesto fundamental. Esta conciencia, captada evidente e indubitablemente, es además el suelo desde el que Husserl considera que se ha de emprender la tarea de una filosofía como ciencia. Esta primacía de un fundamento firme del conocimiento es lo que motiva a Husserl para que asuma como incontrovertible y evidente en el análisis fenomenológico la realidad de la conciencia, cuando de hecho tal evidencia no se desprende de una consideración estrictamente fenomenológica de los datos. Es por esto también que Husserl no aplica una epojé completamente radical, evitando "poner entre paréntesis" la existencia de la conciencia como ente unitario. Hacer esto supondría quedarnos sin base para aclaración radical del conocimiento. Para Husserl, el motivo de la fundación radical del conocimiento prevalece sobre el de la suspensión de toda creencia; por ello, la epojé sólo se ha de aplicar para hacer posible la crítica del conocimiento. Si se aplicara la epojé a la conciencia entonces no habría base desde la que reconstruir la teoría del conocimiento ${ }^{6}$.

Por el contrario, Patočka afirma que una epojé radical, que no excluya la conciencia, conduce a una aclaración más radical del aparecer del mundo. En efecto, lo que se deja ver una vez se suspende la creencia en la realidad incontrovertible de la conciencia es que ésta se encuentra necesariamente arraigada en un mundo, y que éste, lejos de estar "constituido" por la conciencia, es condición de posibilidad de la misma. Un análisis del aparecer que no lo tome por evidencia de una subjetividad transcendental (análisis que es posibilitado por la epojé radical) llega a la conclusión de que la esfera fenomenal se deja describir no como ego-cogito-cogitatum, sino como un sum, como una subjetividad, donde el sujeto se abre al mundo, por mediación de la corporalidad, dándose una continuidad entre el yo y el mundo ${ }^{7}$. El aparecer no es la constitución del objeto en la subjetividad transcendental, sino el aparecer de los objetos en un

\footnotetext{
${ }^{4}$ Cfr. ibidem, p. 105.

${ }^{5}$ Cfr. ibidem, p. 106.

6 Jan Patočka, "Epojé y reducción", en El movimiento de la existencia humana, pp. 246s.

7 Jan Patočka, "El subjetivismo de la fenomenología husserliana y la posibilidad de una fenomenología 'asubjetiva'", pp. 108s.
} 
mundo. Es cierto que el sujeto es un polo al que queda referido el objeto en su aparecer, pero el objeto se refiere al sujeto dentro de la citada estructura continua mediada por el cuerpo. En consecuencia, el análisis del aparecer, una vez la epojé no se detiene en una subjetividad autoevidente, conduce a ver éste como la manifestación de los objetos en el mundo a la subjetividad, que es corporal y que por medio de esa corporalidad se abre al mundo ${ }^{8}$. Antes de que se nos presente el mundo como "totalidad fáctica", se ha presentado como a priori de la subjetividad el mundo como una "totalidad previa" que constituye la base objetiva desde la cual puede tener lugar la intersubjetividad ${ }^{9}$.

Patočka tiene que mostrar cómo puede aparecerse este mundo como una totalidad y cómo puede afirmarse justificadamente que éste es previo a la subjetividad, teniendo en cuenta que, en el análisis del aparecer, éste, por necesidad del mismo método fenomenológico, no puede sino estar referido a la subjetividad. Patočka afirma que es posible mostrar una "autoatestiguación" de la percepción, por la que ésta puede dar cuenta de sí misma por sí misma, sin que la subjetividad quede afirmada como fundante. Patočka afirma que esta autoatestiguación se hace por caminos "enrevesados y no necesariamente evidentes", lo cual deja ver que él mismo era consciente de esta dificultad ${ }^{10}$. Los intentos de Patočka en este sentido se dejan ver en varios de sus textos, los cuales apuntan siempre a la intersubjetividad y a la corporalidad como elementos esenciales en la mostración del mundo como totalidad previa y como condición de posibilidad de la comunicación intersubjetiva que comparte mundo. Así pues, en todo este desarrollo es una pieza fundamental el papel que asume el cuerpo en el aparecer del mundo. Es esta tematización del rol del cuerpo el que vamos a analizar a continuación.

\footnotetext{
${ }^{8}$ Patočka es consciente de que esto supone, en cierta medida, adherirse a la posición de Heidegger. Sin embargo, Patočka no emprende el camino de una elucidación del sentido del ser, sino que toma este aparecer del mundo a la subjetividad como terreno desde el que aclarar radicalmente el manifestarse, donde el mundo quede explicado en su aparecerse, y donde el rol de la subjetividad -y la intersubjetividad- quede aclarado, no ya como mónada, sino como mediación corporal del aparecer del mundo objetivo e independiente.

9 Jan Patočka, "La phénoménologie, la philosophie phénoménologique et les Méditations Cartésiennes de Husserl", p. 159.

10 Jan Patočka, "Universo y mundo del hombre. Anotaciones a un planteamiento cosmológico contemporáneo", en El movimiento de la existencia humana, pp. 85-92.
} 


\section{Corporalidad e intersubjetividad "LocalizadA" en El APARECER DEL MUNDO}

El análisis del cuerpo tiene lugar en varios lugares dentro de la obra de Patočka. En primer lugar, como hemos indicado, la corporalidad es mediación en el aparecer del mundo. La esfera fenomenal no se deja describir como una constitución del mundo en la subjetividad transcendental, sino como aparecer de mundo a través de la corporalidad vivida. Husserl llegó a entrever en La crisis de las ciencias europeas y la fenomenología trascendental que el aparecer es el darse del mundo en la mediación de la corporalidad, pero no llegó a sacar las últimas consecuencias de estos nuevos descubrimientos, permaneciendo en el idealismo ${ }^{11}$. Así pues, el cuerpo vivo es la mediación del aparecer del mundo, donde éste se hace presente en toda su densidad. El hecho de que Husserl no asumiera con todas sus consecuencias el papel del cuerpo vivo (por más que tratara el tema y atisbara que esta cuestión podía hacer estallar el esquema transcendental) se debe a que Husserl no se desprende de una tradición que arranca con Descartes. Esta tradición insiste en obviar los elementos referidos a la corporalidad vivida en el estudio de la subjetividad ${ }^{12}$. En la consideración de la subjetividad, sólo se asumen como notas propias las más "espirituales", mientras que todo lo referido a las vivencias del cuerpo se asimilan sin más al mundo exterior. El empirismo rechaza la sustancialidad de la conciencia subjetiva, pero opera con la misma consideración de la misma como ámbito puramente "mental". En realidad, los empiristas asimilan igualmente la dicotomía entre conciencia y mundo material, por la que niegan la sustancialidad de uno de los términos, explicándola como configurada por los mismos elementos que el resto del mundo. De este modo, afirma Patočka, obvian igualmente la realidad del cuerpo vivo. Kant incide en el mismo olvido del cuerpo vivo, pues recupera ciertamente el papel de la subjetividad como ámbito imprescindible para explicar el fenómeno, pero sigue considerando ésta como una pura estructura intelectual. Llamativamente, sólo hay un autor que Patočka toma como excepción, Maine de Biran. Éste analiza el ego, y ve como rasgo fundamental el que-

11 Jan Patočka, "Epojé y reducción", pp. 246s.

$12 \mathrm{Si}$ no erramos, estas consideraciones, sumamente importantes para comprender el papel del cuerpo vivo en Patočka -que en su pensamiento son vía justo para una nueva objetividad y no para una subjetivización idealista, como veremos- por desgracia no están disponibles aún en idiomas normalmente accesibles. Se encuentran en un curso dictado por Patočka en la Univerzita Karlova de Praga, cuyas transcripciones se han editado en el original checo: Tĕlo, společenství, jazyk, svět [Cuerpo, comunidad, lenguaje, mundo], Praga, Oikoymenh, 1995. 
rer, que se manifiesta como poder. El poder, a su vez, sólo puede hacerse presente en una realidad corporal. En consecuencia, el yo, definido como poder, sólo puede ser tal si es corporal ${ }^{13}$.

El cuerpo vivido es, pues, el medio por el que el mundo se manifiesta en su radical intersubjetividad y espacialidad. Estas dos dimensiones aparecen, según Patočka, conjuntamente y se presuponen mutuamente. La espacialidad se hace presente según la estructura de las tres personas gramaticales, yo-tú-ello (játy-ono). A la subjetividad propia que es el "yo" le corresponde una esencial localización: el "yo" representa el "dentro" originario, el estar en el espacio en un lugar determinado. El "yo" a su vez se encuentra confrontado con un "tú" que representa la radical cercanía, al par que diferencia y oposición. El "tú" está también esencialmente localizado en un lugar que está "ante" el "yo". Estas localizaciones son reconocibles, y los lugares que ocupan pueden cambiar de significado (el lugar que ocupa el tú puede ser ocupado por mí y lo que se presenta como "tú" puede ocupar mi puesto, intercambiándose). Al "yo" y al "tú" se les aparece correlativamente el horizonte del "eso" (ono; en francés, ça), de la tercera persona, que es el que determina al yo y al tú como integrados en una espacialidad. A la vez, el lugar que en un momento se presenta como lugar del "tú" puede dejar de presentarse en absoluta cercanía y pasar a la impersonalidad del "eso", y un lugar que era indiferente puede emerger del horizonte de la tercera persona y presentarse con la radical cercanía del tú ${ }^{14}$. Así pues, la espacialidad del mundo de la vida, primordial respecto del espacio descrito por la geometría, se organiza en el juego intersubjetivo que esencialmente se vincula a diferentes localizaciones, significadas por personas gramaticales ${ }^{15}$.

Este análisis de la espacialidad guarda, a nuestro juicio, gran importancia dentro del intento de Patočka de una fenomenología asubjetiva. Como afirma Marc Richir, si el estudio de la temporalidad lleva a algún tipo de subjetividad constituyente, el estudio de la espacialidad como dimensión fundamental de la esfera fenomenal abre a un ámbito asubjetivo ${ }^{16}$. La espacialidad, en efecto, es

${ }^{13} \mathrm{Cfr}$. Tělo, společenství, jazyk, svět, pp. 23s.

${ }^{14}$ El análisis continúa, explicando Patočka cómo se configura el "nosotros" a partir de la asociación del "yo" con el horizonte del "esto", de modo que se configura el ámbito compartido de desarrollo de la vida. Esta referencia a la constitución de la espacialidad va encaminada a hacer ver el rol que Patočka da a la corporalidad en el aparecer del mundo. 56.

${ }^{15}$ Jan Patočka, "L'espace et sa problématique", en Qu'est-ce que la phénoménologie?. Cfr. pp.45-

\footnotetext{
${ }^{16}$ Cfr. Marc Richir, "Préface", en Jan Patočka, Qu'est-ce que la phénoménologie?, pp. 5-11.
} 
para Patočka una dimensión fundamental del aparecer del mundo, tanto como la temporalidad. De este modo, el estudio del aparecer no conduce a una esfera de conciencia inmanente que se dejaría describir como un fluir temporal, remitido a una comunidad de sujetos, y al fin a un ego, sino a un ámbito espacial previo, compartido con otros sujetos, que se dan con el mismo aparecer del mundo, desde la localización inherente al situarse corporal, la cual queda estructurada según los pronombres personales. En este mundo así abierto espacial e intersubjetivamente, hay aparición sin que propiamente haya "una" subjetividad constituyente ${ }^{17}$.

Patočka estudia detenidamente el papel del cuerpo en el aparecer de la espacialidad originaria $y$, en definitiva, en el aparecer del mundo como totalidad previa. La corporalidad media la aparición del mundo como totalidad, en su espacialidad, como corporalidad vivida y como corporalidad objetiva. El cuerpo vivido, como acabamos de ver, es el que determina la radical localización del sujeto, que abre la espacialidad. Por otro lado, Patočka señala que para que sea posible la aparición del "tú", especialmente en la forma de otro ego, es necesaria la aparición del cuerpo como cuerpo-objeto. En la sola esfera inmanente del ego no puede aparecer como experiencia propia el otro como tal, pues según Patočka, se trataría en última instancia de una experiencia del yo, sin auténtica alteridad. Por otro lado, para que el otro, en cuanto otro, pueda realmente aparecer, hace falta que haya una cierta continuidad. Esta continuidad, que al tiempo es medio del aparecer del otro, como realmente otro, ha de ser una continuidad no subjetiva, pero directamente relacionada con la subjetividad vivida. Este tipo de continuidad la encuentra Patočka en el cuerpo como objeto, éste se presenta en la vivencia, pero se presenta como diferente del cuerpo vivido. Así pues, el cuerpo como cuerpo-objeto es el que abre a experimentar al otro como otro ${ }^{18}$.

17 Si nuestra interpretación es correcta, el estudio de la espacialidad constituye la exposición más completa de un aparecer asubjetivo del mundo. Otros estudios posteriores, pero dentro del mismo periodo final de la vida de Patočka, partirán de la base de este mundo de la vida que aparece asubjetivamente (a cuyos análisis, aquí presentados, dedicará algunas líneas al principio de los ensayos). Desde esta base, proseguirán en una descripción fenomenológica del mundo de la vida, cuyo desarrollo más elaborado y más propio de Patočka es la teoría de los movimientos de la existencia, donde cristaliza su postura antropológica fundamental, que a su vez dará consistencia a sus posiciones ético-políticas. Véase, como ejemplo significativo, "El mundo natural y la fenomenología", en El movimiento de la existencia humana, pp. 13-55.

${ }^{18}$ Esta tesis sobre el cuerpo-objeto como presupuesto del aparecer del otro corresponde a una nota de un texto correspondiente a un curso impartido en la Univerzita Karlova de Praga, y que en francés la han titulado como " $L$ ' homme et le monde". Este curso es una introducción a la fenomenología de Husserl, pero en él Patočka añade importantes comentarios críticos que dan importantes pistas de su pen- 
3. CONCLUSIÓN: LA CORPORALIDAD, MEDIACIÓN (INTER)SUBJETIVA DE LA OBJETIVIDAD.

Así pues, el cuerpo es el vector desde el que el mundo aparece como tal, organizado espacialmente, apareciendo a este sujeto desde su cuerpo, sin que haya primacía del sujeto. El cuerpo, además, se presenta también como sustrato objetivo, en cierto modo extraño a la misma corporalidad vivida subjetivamente, y siendo "terreno común" para la comunicación -lo que, a su vez, abre el camino también al aparecer del mundo objetivo.

No obstante, es preciso señalar que en absoluto Patočka se adhiere a una visión "objetivista" del cuerpo. Todo lo contrario, Patočka asume la preocupación de Husserl por el predominio de la razón científico-técnica. La audacia de Patočka consiste posiblemente en que busca superar el objetivismo científicotécnico desde un nuevo concepto de objetividad, más amplio. Este nuevo concepto de objetividad se corresponde con una apertura a la realidad del mundo en toda su riqueza y variedad, mundo que es independiente y previo al sujeto, quien está abierto.

En el plano concreto de su estudio del cuerpo, su audacia reside, a mi juicio, en que es el cuerpo vivido como tal la matriz desde la que se aparece la espacialidad originaria que muestra un mundo no "fundado" en ninguna subjetividad transcendental sino que se autoatestigua y se muestra como a priori de la subjetividad, siendo a su vez el lugar desde el que se muestra el cuerpo como cuerpo-objeto fundamento para la comunicación.

El cuerpo vivido es, en este intento audaz, el que abre el camino para una desubjetivización de la fenomenología husserliana que, al tiempo, evite los peligros del objetivismo científico-técnico. La argumentación de Patočka, que ya hemos dicho que permanece a nivel de tentativa, adolece de tensiones y es criticable en muchos puntos. De lo que no cabe duda, a mi entender, es de su audacia y de su interés. Patočka, con su intento de fenomenología "asubjetiva", es uno de los autores de referencia en uno de los debates, ya viejos, que se presentan en la discusión fenomenológica, que a su vez tiene grandes repercu-

samiento. Cfr. "L'homme et le monde", en Qu'est-ce que la phénoménologie?, pp. 103s, especialmente la nota 6. 
siones en una reflexión filosófica global: la alternativa entre la primacía del objeto y o la del sujeto. 